Apidologie, 1974, 5 (2), 191-195.

\title{
MÉTHODE DE MESURE DE L'ACTIVITÉ DES ABEILLES
}

\author{
Rémy CHAUVIN \\ Laboratoire d'Ethologie expérimentale \\ Université de Paris \\ Mittainville, 78120 Rambouillet
}

Eine Methode zur Messung der Bienenaktivität

\section{SUMMARY}

METHOD OF MEASURE FOR BEES'ACTIVITY

A device to measure the hive activity is described. It cannot discriminate between the outgoing and the incoming bees but this could be worked out easily. The device is based on the use of a very flexible cylindrical tube (stocking), which the bees fly through. The cylinder is related with a recording pen whose moves are proportional to the weight of the bees passing through the cylinder.

\section{RÉSUMÉ}

On décrit un appareil de mesure pour l'activité d'une ruche. Il ne distingue pas entre l'activité des entrantes et des sortantes mais ceci pourrait facilement être réalisé. L'appareil est basé sur l'utilisation d'un boyau cylindrique très souple (bas de femme) à travers lequel passent les abeilles. Le cylindre est relié à un style enregistreur dont les déplacements sont proportionnels au poids des abeilles qui passent à travers le cylindre.

Dans des travaux déjà anciens (Chauvin, 1952, 1963), j'ai examiné le problème de la mesure de l'activité des abeilles qui quittent la ruche ou y reviennent. Quelques auteurs, que j'ai cités à ce propos, se sont également attaqués à ce problème; ils se sont assez vite découragés sans doute car leurs travaux n'ont pas eu de suite. C'est qu'en effet la mesure de l'activité des abeilles présente des difficultés toutes spéciales. Le nombre des butineuses varie d'abord énormément : de quelques dizaines de milliers par jour à quelques centaines; l'appareil enregistreur doit donc avoir une marge de sensibilité très étendue. Mais une caractéristique de l'activité des abeilles est tout particulièrement gênante : c'est celle qui consiste à déposer un enduit de cire, de propolis ou d'autres substances mal définies, sur tous les objets qui se trouvent à leur contact; l'appareil ne doit donc pas présenter de parties en déplacement trop près les unes 
des autres car elles sont promptement engluées et hors de service. D'autre part, quel est l'instrument qui peut résister pendant de nombreux mois au courant d'air à $30^{\circ}$ saturé d'humidité qui s'échappe de la ruche? Tout ceci élimine une foule de dispositifs comme les contacts électriques ou même les cellules photoélectriques qui ne fonctionnent correctement que pendant plusieurs jours puis nécessitent des nettoyages continuels.

J'ai donc pensé réaliser une sorte de balance en forme de galerie cylindrique, par laquelle les abeilles devraient passer en entrant ou sortant de la ruche. Cette galerie est formée d'un bas de femme (obligatoirement de la qualité dite indémaillable, sans quoi les abeilles le perforent en huit jours environ). On prélève dans ce bas (partie inférieure) un cylindre de $20 \mathrm{~cm}$, de sorte que le diamètre du cylindre soit de $6 \mathrm{~cm}$ environ. Le bas est relié aux deux extrêmités à un anneau en plexiglas qui fait lui-même partie du conteneur décrit dans la figure 1. Le bas doit être maintenu ouvert par une spirale de fil étamé pour cadres (longueur de la spirale $14 \mathrm{~cm}$, écartement des spires $1 \mathrm{~cm}$, poids de la spirale $4 \mathrm{~g}$; elle est maintenue en forme par 4. fils soudés le long de 4 génératrices). Un étrier (Fig. 1,D) passe au-dessous du tout et est

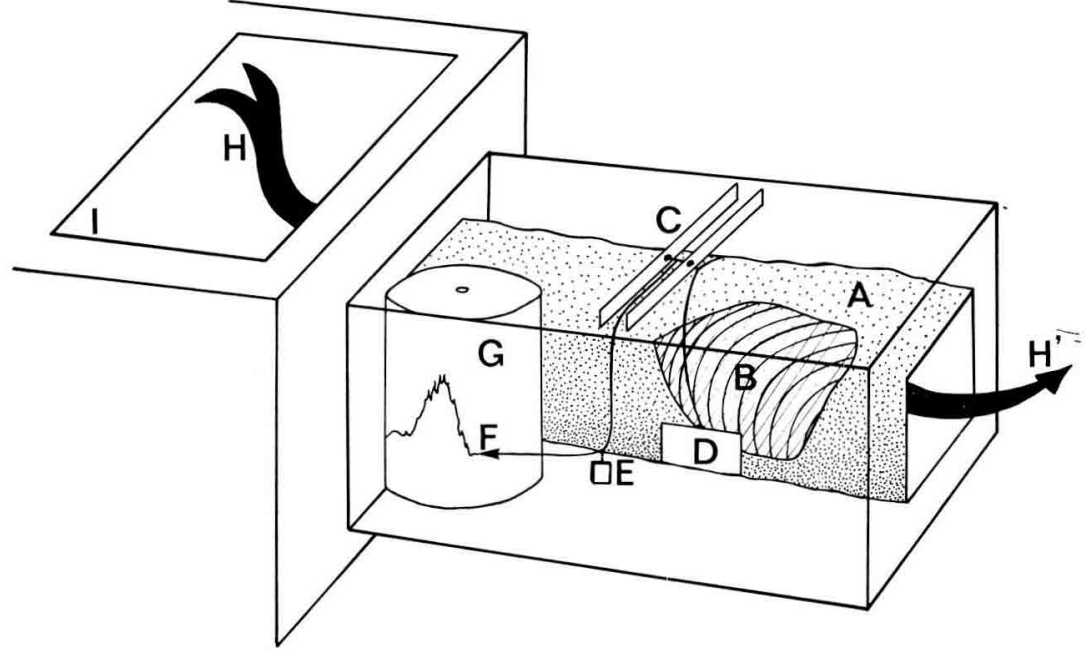

FIG. 1. - Appareil de mesure de l'activité d'une ruche :

A -- Boyau souple à travers lequel passent les abeilles.

B - Armature de fil étamé.

C - Support pour l'axe du style.

D - Étrier du style.

E - Contrepoids.

F - Pointe du style.

$\mathrm{G}$ - Cylindre enregistreur.

H, H' -- Cette flèche montre Ie chemin que suivent les butineuses pour traverser l'appareil.

I - Communication avec la ruche (qui est posée au-dessus de l'ensemble de l'appareil); dimensions de l'ouverture vers la ruche : $10 \times 10 \mathrm{~cm}$.

Aв8. 1. - Apparal zur Messung der Aktivität in einem Bienenvolk :

A - Biegsamer Flugkanal, den die Bienen durchqueren müssen.

B - Versteifendes Gestell aus verzinntem Draht.

C - Halterung für die Schreibfeder-Achse.

$\mathrm{D}$ - Bügel der Schreibfeder.

E - Gegengewicht.

F - Federspitze.

G - Registrierzylinder.

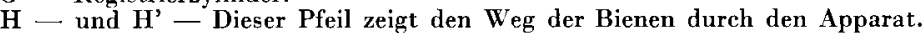

I - Verbindung zum Bienenstock, der sich über dem ganzen Apparat befindet. Die Öffnung beträgt $10 \times 10 \mathrm{~cm}$. 
relié au fléau d'un style inscripteur. Une courbe s'inscrit alors sur un cylindre enregistreur suivant les variations du passage des abeilles à travers le cylindre (longueur des bras du style $3 \mathrm{~cm}$, côté cylindre, et $8 \mathrm{~cm}$ côté enregistreur; contrepoids de $5 \mathrm{~g}$ ).

\section{SENSIBILITÉ DE L'APPAREIL}

Pour un poids de $10 \mathrm{~g}$ l'aiguille du style montre une dénivellation de $15 \mathrm{~mm}$, c'est-à-dire que le passage d'une abeille isolée ne peut évidemment pas être décelé par l'appareil et que le passage de 10 abeilles ne donne lieu qu'à une déviation d'un peu plus de $1 \mathrm{~mm}$. Pratiquement, je considère qu'étant données les pertes occasionnées par le frottement du style contre le papier noirci qui recouvre l'enregistreur, on ne peut guère déceler que 25 abeilles au minimum. Mais étant donné l'énorme quantité des butineuses qui peuvent passer dans le cylindre un jour de miellée, on s'aperçoit qu'une telle précision est bien suffisante et peut-être même inutile.

J'ajouterai que, pendant les jours de grande chaleur succèdant à une miellée, les abeilles se suspendent en grappe dans l'appareil. Il est évidemment bloqué à ce moment; j'étudie un dispositif d'aération permettant d'éviter cet inconvénient.

\section{PREMIERS RÉSULTATS}

L'appareil a été essayé après de multiples remaniements qui ont duré trois ans, sur une ruche Dadant 10 cadres de force moyenne du 22 juillet au 3 août 1973. On a comparé l'inté-

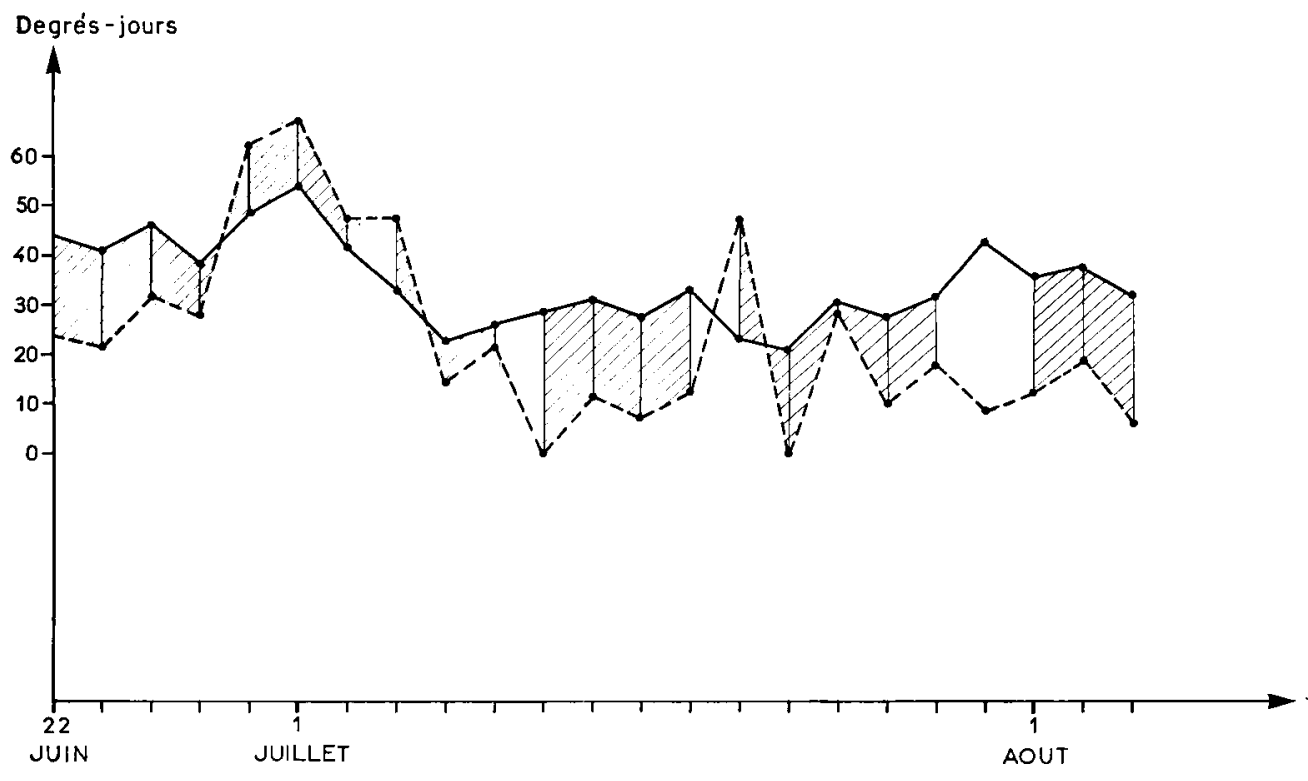

Fıc. 2. - Corrélations entre les degrés-jours (courbe en traits pleins) et l'activité des abeilles (courbe pointillée). Les degrés-jours sont obtenus suivant la méthode classique, en choisissant comme origine la ligne de $10^{\circ} \mathrm{C}$ sur la feuille du thermomètre enregistreur et en pesant la surface de papier comprise entre cette ligne et la courbe des températures pour un jour déterminé. L'activité des abeilles est intégrée de la même manière en pesant la surface de papier comprise entre une ligne d'origine fixe et la courbe décrite par le style enregistreur. Les corrélations positives sont signalées par les hachures.

AвB. 2. - Korrelationen zwischen den Tageswerten (ausgezogene Kurve) und der Bienentätigkeit (unterbrochene Kurve). Die Tageswerte werden nach der klassischen Methode gewonnen, wobei die $10^{\circ} \mathrm{C}-$ Linie auf dem Temperaturschreiber als Ausgang genommen wird und die Fläche zwischen dieser Linie und der Temperaturkurve eines bestimmten Tages in betracht kommt. Die Bienentätigkeit wird auf die gleiche Weise festgestellt, indem man die Fläche zwischen einer fixierten Ausgangslinie und der vom Selbstschreiber aufgezeichneten Kurve in betracht zieht. Die positiven Korrelationen sind durch Schraffierungen angezeigt. 


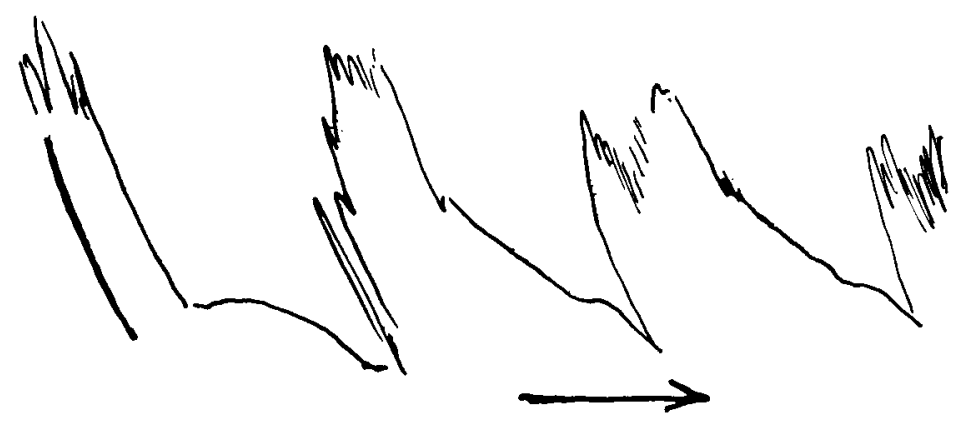

Fıg. 3. - Exemples de courbes d'activité : 28, 29, 30 juin et $l^{\text {er }}$ juillet 1973. Plus de 270 abeilles se trouvent alors en transit dans l'appareil pendant plus de 5 heures.

ABB. 3. - Beispiele von Aktivitätskurven : 28. 29. 30. Juni und 1. Juli 1973. Úber 270 Bienen werden demnach während mehr als 5 Stunden beim Durchgang durch den Apparat angetroffen.

gration des courbes d'activité obtenues en découpant et en pesant la surface recouverte par une courbe journalière, avec les « degrés-jours », intégration des courbes de température d'un thermomètre enregistreur obtenue de la même façon. On observe une très bonne corrélation portant sur 17 jours sur 23 . Une très grosse discordance le 24 août n'a pas été expliquée, ayant été constatée seulement lors du dépouillement des données de septembre.

\section{CONCLUSION}

On projette d'appliquer cette méthode de mesure de l'activité à plusieurs ruches en 1974. Mais il est évident qu'elle pourrait encore subir de nombreux perfectionnements, spécialement en ce qui concerne l'enregistrement. Je n'ai voulu décrire ici qu'un capteur fort bien adapté aux ruches et qui, je l'ai vérifié, ne nuit pas à leur récolte ni à leur développement. Mais il est évident qu'on pourrait adapter à ce capteur un appareil de mesure plus élaboré que celui que j'ai cru devoir choisir à cause de sa simplicité et de sa robustesse.

\section{Reçu pour publication en fëvrier 1974. \\ [Eingegangen im Februar 1974.}

\section{ZUSAMMENFASSUNG}

Der Apparat, mit dem die Aktivität eines Bienenstockes gemessen werden soll, ist in der Hauptsache ein biegsamer Flugkanal, der aus einem Stück maschenfesten Damenstrumpfes besteht. (Nur maschenfestes Material hält den Bienen stand). Die Arbeiterinnen, die den Kanal beim Abflug oder Heimflug durchqueren, werden mit Hilfe eines Gegengewichtes gewogen und die jeweiligen Werte auf einem zylindrischen Selbstschreiber aufgetragen. Mindestens 25 Bienen sind für einen Ausschlag der Schreibfeder notwendig. Dadurch erhält man gute Korrelationen zwischen der Aktivität der Sammelbienen und der Aussentemperatur wesentlich bessere, als sie von früheren Autoren aufgezeigt wurden. Fine solche Fangvorrichtung kann verschiedene und vollkommenere Registrierungen liefern. Zudem erlaubt das Verfahren, den Apparat während des ganzen Bienenjahres auf einer 10 Waben-Dadantbeute mit normal starkem Volk in Betrieb zu halten, ohne dass der Apparat gereinigt werden müsste oder die Volksentwicklung gestört würde. Das konnte bisher mit keinem Verfahren erreicht werden. 


\section{REFERENCES BIBLIOGRAPHIQUES}

Chauvin R., 1952. Nouvelle technique d'enregistrement de l'activité de la ruche. L'Apiculteur (Sec. Scientifique), 96 (3), 9-14.

Chauvin R., 1963. Essai d'enregistrement simultané des principaux phénomènes de la vie d'une ruche. Ann. Abeille, 6, 167-183. 